\section{First edible cottonseed go-ahead}

US regulators in October approved a transgenic cotton plant that produces edible seed. The plant, developed by Texas A\&M AgriLife Research in College Station, is genetically engineered to greatly reduce the expression of the antinutrient gossypol in its seed, making it edible for humans and animals. Gossypol is a naturally occurring plant pigment produced in the glands of cotton that is toxic to insects and serves as a natural defense mechanism against pests. The pigment can also be toxic to humans and most monogastric animals, such as pigs, birds, fish and rodents, when consumed at high levels. Keerti Rathore and his colleagues at Texas A\&M modified the cotton (Gossypium hirsutum) by silencing, with RNA interference, the genes in the seed that encode $\delta$-cadinene synthase, a key enzyme in gossypol biosynthesis. This lowers gossypol in the seed by $97 \%$, to 300 parts per millionbelow the US Food and Drug Administration's safety threshold. The rest of the cotton plant maintains normal levels of gossypol, enabling the plant to keep its inherent pest defenses. The technology could increase the value of cottonseed to farmers and others along the commercial chain, and provide a new protein source for humans and animals, Rathore says. The cotton plant triggered regulatory oversight by the US Department of Agriculture (USDA) because it was transformed using Agrobacterium tumefaciens, which the agency considers a "plant pest." The agency in August also approved for commercialization genetically engineered canola seed with increased levels of docosahexaenoic acid (DHA), an omega-3 fatty acid, developed by Nuseed in Breckenridge, Minnesota. Meanwhile, the USDA has increasingly given free passes to plants genetically altered using CRISPR, enabling the plants to be commercialized without the agency's oversight (Nat. Biotechnol. 36, 6-7, 2018). That includes, most recently, a camelina line from Yield10 Bioscience in Woburn, Massachusetts, and a pennycress line from Illinois State University in Normal, Illinois, both with undisclosed phenotypes.

Emily Waltz

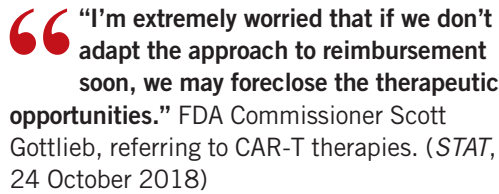

"We should expect Al merely to try its best to give us exactly what we ask for, and we should be very careful what we ask for." Janelle Shane comments on the darker side of machine learning. (The New York Times, 28 October 2018) helps extend the survival of men with prostate tumors that have metastasized there. Bayer of Leverkusen, Germany, initially joined with the drug's originator, Algeta, to run phase 3 trials for Xofigo. But after the drug's approval in 2013, Bayer bought its partner outright for nearly $\$ 3$ billion-and Bayer continues to be active in radionuclide drug development today.

Novartis's foray into radiopharmaceuticals is not recent: it began in the late 1990s, when it coupled its drug Sandostatin (octreotide), a somatostatin analog, to a radioactive warhead. Sandostatin had been used for decades to block hormone release in endocrine tumors. With the yttrium-90 ions, the drug-called 90Y-DOTATOC or OctreoTher-did more than block somatostatin receptors; it actively destroyed the types of neuroendocrine tumors that express these receptors on their cell surfaces.

After testing OctreoTher in more than 300 patients-around one-quarter of whom responded to the therapy-Novartis filed for marketing consideration with the FDA. The agency said no, though. According to David Bushnell, a nuclear medicine doctor at the University of Iowa Carver College of Medicine in Iowa City who led trials with the drug, the FDA wanted to see randomized clinical data before granting approval.

Novartis opted instead to offload the drug on Molecular Insight Pharmaceuticals, a company with a radionuclide therapy called Azedra (iobenguane I 131) in phase 1 testing at the time. Molecular Insight was acquired in 2013 by Progenics Pharmaceuticals; and in July 2018, Azedra-a conjugate of iodine-131 with an norepinephrine analog-won FDA approval for treating two types of rare adrenaline-producing neuroendocrine cancers. OctreoTher never advanced much further in these companies' hands.

Novartis only returned to radiotherapeutics a decade later, after AAA did the heavy lifting for them. AAA tested Lutathera in a randomized trial in patients with metastatic midgut neuroendocrine tumors. Compared with the control arm, Lutathera dramatically extended progression-free survival with no evidence of kidney damage thanks to the administration of protective amino acids (N. Engl. J. Med. 376, 125-135, 2017).

"That's fairly unprecedented," says Jonathan Strosberg, a neuroendocrine tumor specialist at the Moffitt Cancer Center in Tampa, Florida, who led that phase 3 trial. Those data formed the basis of the FDA's approval, in January 2018, for Lutathera to treat neuroendocrine tumors of the gastrointestinal tract and pancreas.

Lutathera also couples a somatostatin ana$\log$ to an isotope that emits high-energy beta particles. But instead of yttrium-90, Lutathera uses lutetium-177, a lower energy isotope that avoids some of the collateral damage to neighboring tissues. Lutetium-177 also has a longer half-life-about 6.6 days, compared with 2.7 days for yttrium-90-which means it "can be prepared centrally and distributed ready to use, avoiding uncertainties and logistics of onsite preparation," says Ballinger.

Plus, since lutetium-177 emits low levels of gamma rays on top of its higher energy beta rays, Lutathera can be tracked inside the body via single-photon emission computerized tomography. "You can watch the thing work," says Michael Tweedle, of the Ohio State University in Columbus, who, as president of Bracco Research USA, previously helped develop a lutetium-177-labeled drug for targeting gastrin-releasing peptide receptors on tumor cells. "It allows you get dosimetry data on a patient-by-patient basis," he says.

Lutetium-177 also underpins Endocyte's lead candidate, 177Lu-PSMA-617, which pairs the isotope with a small molecule designed to bind prostate-specific membrane antigen (PSMA), a protein highly expressed on the surface of prostate cancer cells. The drug was developed by ABX of Radeberg, Germany, and licensed in October 2017-but much of the PSMAtargeting technology actually comes from the laboratory of Philip Low, director of the Purdue University Institute for Drug Discovery in West Lafayette, Indiana, who founded Endocyte in the mid-1990s and has served as the company's CSO for the past 20 years.

An earlier PSMA-targeted small-molecule developed by Endocyte, EC1169, had, however, failed to shrink tumors in phase 1 testing. The agent, a microtubule inhibitor paired to a targeting ligand, zeroed in on prostate tumor cells as it was supposed to, but once there, didn't seem to release the therapeutic cargo.

Fortunately, the targeting part of the radiolabeled agent 177Lu-PSMA-617, which Low describes as "almost identical" to Endocyte's earlier compound, shouldn't suffer the same issue. "All you have to do is locate the lutetium near the cancer cell; you don't have to release it within the cancer cell," says Low. "That distinction simplifies the therapeutic mechanism enormously." Endocyte's 750-person, phase 3 VISION study for 177Lu-PSMA-617 began enrolling patients earlier this year. Other radiolabeled products in pivotal testing are Actinium Pharmaceuticals' Iomab-B, which directs lutetium-177 to CD45-expressing B cells in follicular lymphoma; and Nordic Nanovector's 177Lu-lilotomab satetraxetan (Betalutin), which uses iodine-131 to destroy the bone marrow ahead of stem cell transplantation (Table 1). 